\title{
Integrated Optical Device for Frequency Conversion Across the Full Telecom $C$-Band Spectrum
}

\author{
Paul Fisher®, ${ }^{1, *}$ Matteo Villa, ${ }^{1}$ Francesco Lenzini, ${ }^{1,2}$ and Mirko Lobino $\odot^{1,3, \dagger}$ \\ ${ }^{1}$ Centre for Quantum Computation and Communication Technology (Australian Research Council), \\ Centre for Quantum Dynamics, Griffith University, Brisbane, QLD 4111, Australia \\ ${ }^{2}$ Institute of Physics, University of Muenster, Muenster 48149, Germany \\ ${ }^{3}$ Queensland Micro- and Nanotechnology Centre, Griffith University, Brisbane, QLD 4111, Australia
}

(Received 9 July 2019; revised manuscript received 29 October 2019; accepted 24 December 2019; published 10 February 2020)

\begin{abstract}
High-density communication through optical fiber is made possible by wavelength-division multiplexing (WDM), which is the simultaneous transmission of many discrete signals at different optical frequencies. Vast quantities of data may be transmitted without interference using this scheme but flexible routing of these signals requires an electronic middle step, which carries a cost in latency. We present a technique for frequency conversion across the entire WDM spectrum with a single device, which removes this latency cost. Using an optical waveguide in lithium niobate and two infrared pump beams, we show how to maximize the conversion efficiency between arbitrary frequencies by analyzing the role of dispersion in cascaded nonlinear processes. The technique is presented generally and may be applied to any suitable nonlinear material or platform and to classical or quantum optical signals.
\end{abstract}

DOI: 10.1103/PhysRevApplied.13.024017

\section{INTRODUCTION}

Optical communications have greatly increased the data capacity of telecommunication networks since signals can be transmitted faster and with a greater bandwidth compared to copper lines and can be sent in multiple streams over one fiber using wavelength-division multiplexing (WDM). The lowest propagation losses through optical fiber are in the $C$ band, which supports a standard grid of 72 channels spaced by $100 \mathrm{GHz}$ between $1520 \mathrm{~nm}$ and $1577 \mathrm{~nm}$ [1]. The transfer of signals between these channels represents a major speed limitation of current networks, since this operation is performed by terminating and measuring all signals with a bank of passive optics and detectors and then retransmitting them using a bank of lasers [2]. All-optical signal processing can overcome this speed bottleneck, since it can convert data streams between different channels almost instantaneously. In particular, having a single device that can convert data between arbitrary wavelengths without interruption will make a huge impact on current telecommunications technology.

\footnotetext{
*paul.fisher@griffithuni.edu.au

†m.lobino@griffith.edu.au
}

Published by the American Physical Society under the terms of the Creative Commons Attribution 4.0 International license. Further distribution of this work must maintain attribution to the author(s) and the published article's title, journal citation, and DOI.
There are a number of existing devices for routing WDM signals, known as reconfigurable optical add-drop multiplexers (ROADMs). They have been implemented in a number of platforms, including micromachined reflectors [3] and thermally tuned microfabricated resonators [4]. Although technically flexible, these devices physically route signals without altering the wavelength and are limited to narrow bandwidths or static grid spacing. To switch channels dynamically and leave physical routing to a static structure, uninterrupted channel swapping can be achieved by frequency conversion using nonlinear optics.

Nonlinear optical schemes for frequency conversion over the telecom $C$ band have focused primarily on fourwave mixing in $\chi^{(3)}$ nonlinear materials such as singlemode [5] and photonic crystal fibers [6,7] and silicon waveguides [8]. These schemes suffer from the inherently small value of the $\chi^{(3)}$ coefficient, requiring long fibers and high pump powers, or high-quality resonators that reduce the tuning bandwidth. More efficient conversion techniques, such as sum and difference frequency generation (SFG and DFG, respectively) in $\chi^{(2)}$ nonlinear materials such as lithium niobate, have already demonstrated high conversion efficiency in small $(5-\mathrm{cm})$ devices using modest (90 $\mathrm{mW} \mathrm{cW}$ ) pump powers [9]. To achieve the frequency shift between two WDM channels, however, these two processes must be cascaded, resulting in an overall interaction that mimics a $\chi^{(3)}$ process.

Cascading can be performed with SFG and DFG steps occurring sequentially, either in two waveguides [10] or 
in opposite directions in the same waveguide [11]. More commonly, cascading refers to performing the SFG and DFG steps simultaneously with two pump lasers, as in Fig. 1. Initially, it was proposed as an alternative method to single-step DFG to achieve frequency shifts. Bright pumps generate second-harmonic or sum-frequency light, which subsequently generates a difference frequency with an input signal. Demonstrations have been performed $[12,13]$ and the technique has been analyzed in several configurations [14-16] and in comparison to single-step DFG [17]. Unfortunately, this also results in parametric amplification of the input frequency, which is not suitable for signal dropping.

In an alternative pump configuration, cascading can emulate degenerate four-wave mixing for conversion and signal dropping. In this case, one pump converts the signal by SFG to an intermediate frequency, which is then converted to the target frequency by DFG with a second pump. Experiments have verified this technique in $\mathrm{cw}$ and pulsed-pump regimes $[18,19]$ and have shown its suitability for telecom signals [20]. Subsequent experiments have attempted to improve the conversion bandwidth [21,22] or enhance signal dropping and selectivity through engineered poling [23] and thermal gradients [24]. However, these devices have a limited operational bandwidth and cannot efficiently convert between any arbitrary pair of channels.

Here, we propose an optimized protocol for highefficiency frequency conversion across the entire WDM spectrum and demonstrate it using a waveguide fabricated in periodically poled lithium niobate. We overcome the limitation of previous schemes by analyzing the role of phase mismatch and finding the optimal configuration

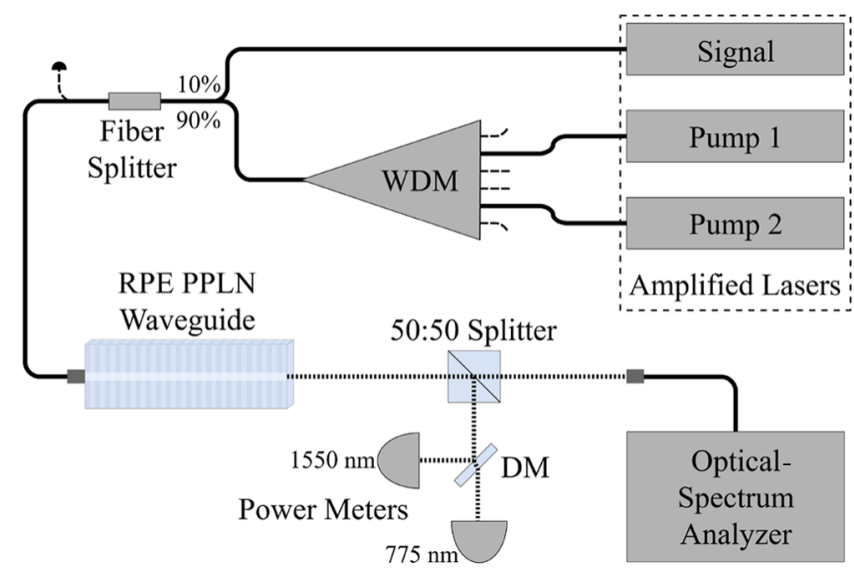

FIG. 1. A schematic of the experimental setup. A WDM module combines the two pump lasers while the signal is added with a 90:10 fiber coupler. All of the components are then coupled to the waveguide. Light is collected from the waveguide in free space, with half diverted to an optical-spectrum analyser and the rest collected by two power meters across a dichroic mirror (DM). when using a uniform periodic poling for quasi-phasematching (QPM). In particular, we show that with reverseproton-exchanged waveguides in lithium niobate, maximum tunable conversion across the entire telecom $C$ band can be achieved using pumps around $2.38 \mu \mathrm{m}$. We demonstrate our protocol and show agreement between theory and experiment, performing frequency-conversion measurements using pumps also within the $C$ band. While this is not the optimal pump configuration, we achieve over $30 \%$ conversion and over $80 \%$ signal dropping in a waveguide a with $3.2 \mathrm{~cm}$ interaction length.

The impact of these results goes beyond classical communication and can be applied to quantum networks as well. In particular, our device can be used to interface narrow-bandwidth erbium quantum memory [25] with all the WDM channels, greatly increasing the capacity of future quantum repeater networks. Furthermore, this protocol may be used where four-wave mixing has been applied in the past, with enhancement of the heralding rate and purity of a spontaneous parametric down-conversion single-photon source being an example [26].

\section{CASCADED FREQUENCY CONVERSION}

We now derive the conditions for efficiently converting a signal data stream encoded in any of the WDM channels at frequency $\omega_{s}$ into a target frequency $\omega_{t}$ using a single periodically poled nonlinear waveguide. The first step is SFG between the signal $\omega_{s}$ and a pump beam $P_{1}$ at $\omega_{P 1}$, which generates a new field at frequency $\omega_{\mathrm{SFG}}=$ $\omega_{P 1}+\omega_{s}$. The second step is DFG between a second pump $P_{2}$ at $\omega_{P 2}$ and the field at $\omega_{\mathrm{SFG}}$. The frequency of the second pump is chosen such that the DFG goes to the desired target frequency $\omega_{t}=\omega_{\mathrm{SFG}}-\omega_{P 2}$. Figure 2(a) shows a schematic of these interactions.

In this protocol, both interactions happen simultaneously in a single nonlinear waveguide where the signal and the two pumps are coupled together. In total, there are five frequency components participating in the conversion, each with a different phase velocity. As a consequence, there are two phase mismatches to consider, given by

$$
\begin{aligned}
\Delta k_{\mathrm{SFG}} & =k_{\mathrm{SFG}}-k_{s}-k_{P 1}, \\
\Delta k_{\mathrm{DFG}} & =k_{\mathrm{SFG}}-k_{t}-k_{P 2},
\end{aligned}
$$

where, for every field involved, $k_{i}=2 \pi n_{\mathrm{eff}, i} / \lambda_{i}$ is the propagation constant of the mode at frequency $\omega_{i}=2 \pi c / \lambda_{i}$, and $n_{\text {eff }, i}$ is its effective refractive index.

In order to derive the conversion efficiency and its bandwidth, it is useful to consider the average $(K)$ and the difference $\left(\delta_{K}\right)$ of the two phase mismatches:

$$
\begin{aligned}
K & =\left(\Delta k_{\mathrm{SFG}}+\Delta k_{\mathrm{DFG}}\right) / 2, \\
\delta_{K} & =\Delta k_{\mathrm{SFG}}-\Delta k_{\mathrm{DFG}} .
\end{aligned}
$$


(a)

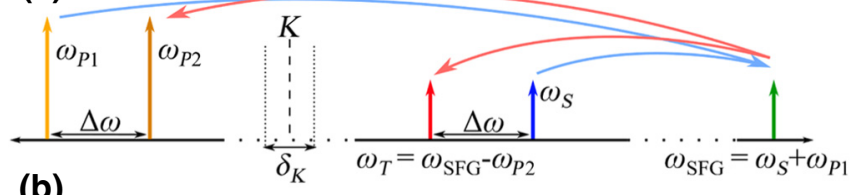

(b)

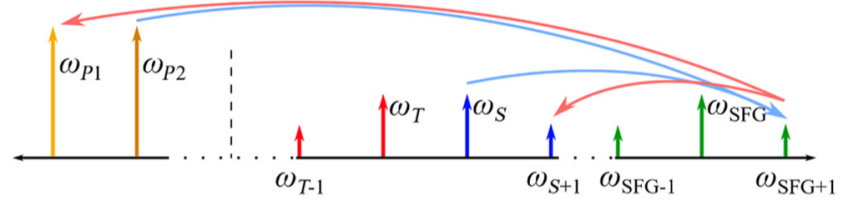

(c)

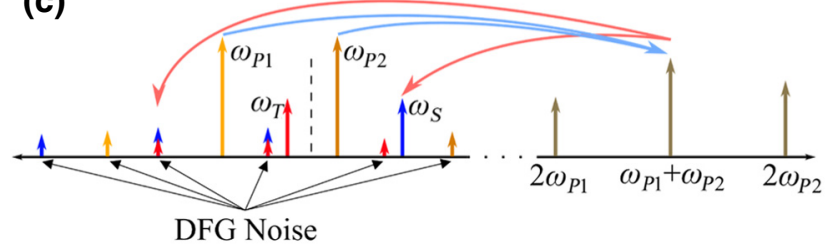

FIG. 2. Nonlinear interactions in the waveguide. (a) The correct SFG and DFG interaction for converting light from $\omega_{s}$ to $\omega_{t}$. First, $\omega_{\mathrm{SFG}}$ is generated by SFG between the signal and pump 1. Then the target frequency $\omega_{t}$ is generated by DFG $\omega_{\mathrm{SFG}}-\omega_{P 2}$. The frequency shift between the signal and the target is $\Delta \omega=\omega_{P 1}-\omega_{P 2}$. (b) Unwanted interaction with the wrong pumps. New frequencies are generated by SFG $\left(\omega_{s(t)}+\right.$ $\left.\omega_{P 2(P 1)}=\omega_{\mathrm{SFG}+1(\mathrm{SFG}-1)}\right)$ and subsequent DFG $\left(\omega_{\mathrm{SFG}+1(\mathrm{SFG}-1)}-\right.$ $\left.\omega_{P 1(P 2)}=\omega_{s+1(t-1)}\right)$, (c) Sum-frequency generation between the two pump beams. This new SFG field also interacts with the signal, target, and pumps, and can generate other frequencies by DFG.

The value of $K$ determines the optimal poling period $\Lambda=$ $2 \pi / K$, since it satisfies the QPM condition $K_{\mathrm{QPM}}=K-$ $2 \pi / \Lambda=0$. It can be shown that for conversion between two arbitrary frequencies, the condition $K_{\mathrm{QPM}}=0$ can always be satisfied with the correct choice of the two pump frequencies $\omega_{P 1}$ and $\omega_{P 2}$. The second quantity $\delta_{K}$ is analogous to the phase mismatch of the corresponding four-wave mixing process and is the primary factor that limits the overall conversion efficiency.

When the pump powers are optimized, the conversion $\left(\eta_{c}\right)$ and signal-dropping $\left(\eta_{d}\right)$ efficiencies, the latter being the fraction of power removed from the signal channel, are given by

$$
\begin{aligned}
& \eta_{c}=\frac{Q^{2} L^{4}}{16} \operatorname{sinc}^{4}\left(\frac{1}{4} \sqrt{\delta_{K}^{2} L^{2}+4 Q L^{2}}\right), \\
& \eta_{d}=\frac{Q L^{2}}{2} \operatorname{sinc}^{2}\left(\frac{1}{4} \sqrt{\delta_{K}^{2} L^{2}+4 Q L^{2}}\right)-\eta_{c} .
\end{aligned}
$$

$Q$ is a function of the total pump power and is proportional to the square of the $\chi^{(2)}$ nonlinearity. These efficiencies also depend on the length of the device $L$, illustrating that total phase mismatch $\delta_{K} L$ is cumulative and becomes more detrimental over longer devices. Maximum conversion is achieved when $Q=\pi^{2} / L^{2}$. Therefore, longer devices require less pump power to achieve maximum conversion, so the practicalities of available pump powers and damage thresholds must be weighed against tolerance to phase mismatch.

The pump powers are optimized when they are balanced by the ratio

$$
\frac{P_{1}}{P_{2}}=\frac{\omega_{t} n_{\mathrm{eff}, s} n_{\mathrm{eff}, P 1} A_{\mathrm{eff}, \mathrm{SFG}}}{\omega_{s} n_{\mathrm{eff}, t} n_{\mathrm{eff}, P 2} A_{\mathrm{eff}, \mathrm{DFG}}}
$$

where $A_{\mathrm{eff}, i}$ are the effective areas of the SFG and DFG processes inside the waveguide. This ensures that the processes progress at the same rate as one another and that the only factor unbalancing them is the phase mismatch. For a detailed derivation of this protocol and all of the introduced quantities and formulas, see the Supplemental Material [27].

Other factors to be considered in the design of a device relate to the suppression or minimization of unwanted nonlinear processes. Because there are five different frequencies propagating inside the same waveguide, unwanted three-wave mixing interactions are possible. For example, the signal and target fields may interact with the wrong pumps, generating new frequencies around $\omega_{\mathrm{SFG}}$ and subsequent DFG into the wrong channels [see Fig. 2(b)]. Also, the pumps may interact with one another and generate a sum frequency or second harmonics, which may then produce other unwanted frequencies in the $C$ band by DFG [see Fig. 2(c)].

Unwanted processes can be mitigated or suppressed by ensuring that they are not quasi-phase-matched. Because the phase-matching bandwidth decreases with an increasing interaction length, longer devices reduce these unwanted effects better than shorter ones. The case in which the signal interacts with the wrong pump [Fig. $2(b)]$ and generates the wrong SFG $\bar{\omega}_{\mathrm{SFG}}=\omega_{s}+\omega_{P 2}$ largely depends on the dispersion properties of the device around $\omega_{\mathrm{SFG}}$. While the average phase mismatch $\bar{K}$ of this unwanted process is generally nonzero, greater chromatic dispersion around $\omega_{\mathrm{SFG}}$ means that the magnitude of $\bar{K}$ will be larger and the unwanted process will be less efficient. The pump second harmonics $2 \omega_{P 1}$ and $2 \omega_{P 2}$, and SFG $\omega_{P 1}+\omega_{P 2}$, are easily reduced by choosing the frequencies $\omega_{P 1}$ and $\omega_{P 2}$ away from those of the signal and the target, but this also reduces the conversion efficiency and tuning bandwidth as the magnitude of $\delta_{K}$ tends to increase.

These results are summarized in Fig. 3 where the average conversion efficiency between all pairs of WDM channels, for a total of 5256 combinations, is plotted as a function of the mean pump wavelength $\sum_{n=1}^{5256}\left(\lambda_{P 1_{n}}+\right.$ $\left.\lambda_{P 2_{n}}\right) / 10512$ and the corresponding poling period. The shaded area in the figure represents one standard deviation around the average efficiency and is an indication of 


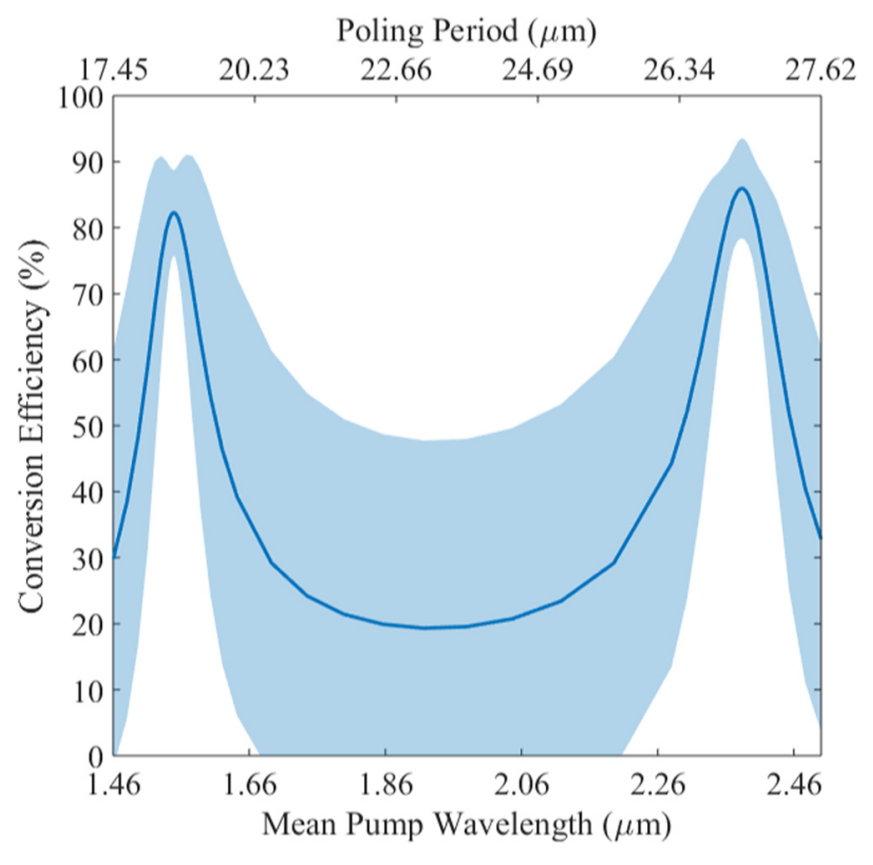

FIG. 3. The mean conversion efficiency over all $C$-band channels (spans 190.0 THz to $197.2 \mathrm{THz}$, minimum step of $100 \mathrm{GHz}$ ), against the average pump wavelength and the poling period. The shaded bars show one standard deviation in the efficiencies of all channels. It includes the effects of propagation loss, wrong-pump interaction and pump SFG/SHG noise.

how broadband the process can be. These data are calculated numerically using the dispersion curve of bulk lithium niobate [28] for a 5-cm-long device with an effective area of $25 \mu \mathrm{m}^{2}$, pumps optimized according to Eq. 7 , and propagation losses of $0.1 \mathrm{~dB} / \mathrm{cm}$, which are standard for reverse-proton-exchanged waveguides. The system of coupled-mode equations includes more modes than the original five to reflect wrong-pump interactions $(+12$ modes) and unwanted SFG/SHG interactions between the pumps (+7 modes). Figure 3 shows that for pump frequencies close to the signals and targets, the conversion efficiency is $82 \%$ on average across the WDM spectrum, as $\delta_{K}$ remains small when the pumps are close to the signals. Ideal conversion $\left(\delta_{K}=0\right)$ is not possible, however, as this occurs when the pump frequencies are equal to the signal and target frequencies.

The most important result of this analysis is that because of the chromatic dispersion of the material, ideal conversion can be obtained with pumps near $2.38 \mu \mathrm{m}$. This is possible because $2.38 \mu \mathrm{m}$ lies on the opposite side of an inflection in the dispersion curve of lithium niobate to $1.55 \mu \mathrm{m}$, allowing $\delta_{K}$ to go to zero. In this case, the conversion efficiency is $86 \%$ on average across the whole WDM spectrum, as shown in Fig. 3. This improvement over the case of telecom pumps is due to the lower dispersion around $2.38 \mu \mathrm{m}$, meaning the $\delta_{K}$ remains smaller across the entire WDM spectrum. Additionally, the entire WDM spectrum is available for use, as the pumps are well separated from the signals. While pumps around $2.38 \mu \mathrm{m}$ give the highest conversion efficiency for lithium niobate, other materials will have different optimal pump wavelengths, depending on their dispersion properties.

The drawback to using $2.38 \mu \mathrm{m}$ pumps is that the effect of interaction with the wrong pump is made worse. The chromatic dispersion around $\omega_{\mathrm{SFG}}$ is smaller than it is for $1.55 \mu \mathrm{m}$ pumps. This makes the average phase mismatch $\bar{K}$ of the unwanted process smaller in magnitude, so the process is more efficient. To illustrate, using the data calculated for Fig. 3, 2.1\% of the signal power is lost to crosstalk for a $200 \mathrm{GHz}$ step using $1.55 \mu \mathrm{m}$ pumps. This loss increases to $7.8 \%$ using $2.38 \mu \mathrm{m}$ pumps for the same $200 \mathrm{GHz}$ step. The cost for mitigating this crosstalk is to fabricate longer devices, which provides a technical challenge, or to make the channels more broadly spaced. The loss to crosstalk drops below $1 \%$ for frequency steps larger than $600 \mathrm{GHz}$.

In data transmission, information is encoded in light pulses that usually have a bandwidth up to $28 \mathrm{GHz}$ for $100 \mathrm{GHz}$ WDM systems. To evaluate the pulse distortion during the conversion process, we evaluate the conversion efficiencies at the sidebands $\pm 14 \mathrm{GHz}$ [20]. When the pump wavelengths are around $1550 \mathrm{~nm}$, the average difference in conversion efficiency between the peak and the sidebands for all 5256 combinations is $\Delta \eta=10.7 \pm 0.3 \%$, where the error is one standard deviation from the mean value. The situation improves when the pumps are in the $2.38 \mu \mathrm{m}$ region with $\Delta \eta=3.03 \pm 0.06 \%$. In both cases, pulse distortion is small.

\section{EXPERIMENTAL RESULTS}

The conversion protocol is used to perform a set of frequency-conversion measurements using the setup of Fig. 1. Pump lasers in the $C$ band are used, resulting in a nonoptimal conversion as shown in Fig. 3. This choice is made because tunable lasers around $2.38 \mu \mathrm{m}$ wavelength, which give higher conversion efficiency, are not available in our laboratory.

The central element of the setup is a nonlinear periodically poled waveguide in lithium niobate, fabricated using the reverse-proton-exchange technique $[29,30]$. The device is $6 \mathrm{~cm}$ long, with a $5-\mathrm{cm}$ poled region of period $\Lambda=$ $16.02 \mu \mathrm{m}$ and nominal propagation losses of $0.1 \mathrm{~dB} / \mathrm{cm}$. The device is also heated to $106^{\circ} \mathrm{C}$ to avoid photorefractive damage at high pump power and to tune the resonance frequencies of the nonlinear processes to be in line with the WDM channels.

To characterize the waveguide and estimate its conversion efficiency, we measure the second-harmonic generation as a function of the pump wavelength and estimate an interaction length of $3.2 \mathrm{~cm}$ with an effective area of the SHG process of $42 \mu \mathrm{m}^{2}$. For details on this measurement, 
TABLE I. A summary of the main results of the frequency-conversion measurements: the wavelengths of the signal, target, and pumps, the pump power levels used, the measured efficiencies, and their theoretical estimations. The power values are measured out of the waveguide and corrected for losses in the measurement setup.

\begin{tabular}{lccccccccc}
\hline \hline$\lambda_{s}(\mathrm{~nm})$ & $\lambda_{t}(\mathrm{~nm})$ & $\lambda_{P 1}(\mathrm{~nm})$ & $\lambda_{P 2}(\mathrm{~nm})$ & $P_{1}(\mathrm{~mW})$ & $P_{2}(\mathrm{~mW})$ & $\eta_{c}^{\text {exp }}$ & $\eta_{d}^{\text {exp }}$ & $\eta_{c}^{\text {th }}$ & $\eta_{d}^{\text {th }}$ \\
\hline 1533.465 & 1531.898 & 1555.021 & 1556.636 & $131.1 \pm 0.9$ & $124.9 \pm 0.9$ & $0.19 \pm 0.01$ & $0.070 \pm 0.004$ & 0.403 & 0.123 \\
1533.465 & 1530.334 & 1555.021 & 1558.254 & $121.0 \pm 0.9$ & $127 \pm 1$ & $0.28 \pm 0.02$ & $0.16 \pm 0.01$ & 0.385 & 0.156 \\
1530.334 & 1535.036 & 1558.254 & 1553.409 & $136 \pm 1$ & $121 \pm 1$ & $0.31 \pm 0.02$ & $0.134 \pm 0.008$ & 0.403 & 0.107 \\
1535.036 & 1528.773 & 1553.409 & 1559.875 & $125 \pm 1$ & $125 \pm 1$ & $0.28 \pm 0.02$ & $0.162 \pm 0.007$ & 0.387 & 0.144 \\
1528.773 & 1536.609 & 1559.957 & 1551.881 & $124.0 \pm 0.9$ & $125.4 \pm 0.9$ & $0.26 \pm 0.02$ & $0.18 \pm 0.02$ & 0.385 & 0.146 \\
\hline \hline
\end{tabular}

see the Supplemental Material [27]. The overall insertion losses of the device are $70 \%$, which gives an estimated coupling loss of $66 \%$.

The two pump lasers are combined into a single fiber using a commercial WDM module with a channel spacing of $200 \mathrm{GHz}$. The pass band of this WDM module allows up to $\pm 40 \mathrm{GHz}$ of tuning around the peak frequencies and different frequency conversions are performed using different WDM channels. The signal beam is combined with the two pumps using a 90:10 fiber coupler, with the pumps coupled into the $90 \%$ arm in order to maximize the amount of pump power available for the experiment.

Five different frequency-conversion experiments are performed with frequency shifts between the signal and the target ranging from 0.2 to $1 \mathrm{THz}$. During the experiments, the output of the waveguide is collected with an achromatic lens and sent to a 50:50 beam splitter. After the beam splitter, light is sent to power meters and an opticalspectrum analyzer (OSA) simultaneously. Data collection is automated and the pump frequencies are scanned in 5 $\mathrm{GHz}$ increments to find the peak conversion efficiency. The pump relative powers are balanced using traces from the OSA for each measurement while the signal power transmitted through the waveguide is always around $1 \mathrm{~mW}$. The values of the wavelengths used, the pump powers, and the measured and calculated efficiencies are summarized in Table I.

Figure 4 shows the OSA traces of the frequency conversion from $\lambda_{s}=1555.021 \mathrm{~nm}$ to $\lambda_{t}=1558.254 \mathrm{~nm}$. From these data, we can see that several parasitic nonlinear effects are present. Figure 4(c) shows that the correct SFG field has the highest peak but second harmonics from the two pumps as well as SFG between the two pumps are also present. The smaller peaks near the SFG one are generated by interaction with the wrong pumps, as shown in Fig. 2(b). Figure 4(a) shows the signal and target peaks and the crosstalk with the other two unwanted channels. In this particular case, the power in the unwanted channels is $16.46 \mathrm{~dB}$ and $25.12 \mathrm{~dB}$ smaller than the power in the target channel. From these traces, it is possible to obtain the relative intensities of all the frequency components and when combined with the total power measured with the power meters, absolute power measurements of each component can be inferred.
The conversion protocol is further tested by measuring the dependence of the converted power as a function of the combined pump powers, with the powers of $P_{1}$ and $P_{2}$ approximately balanced. Power measurements at the
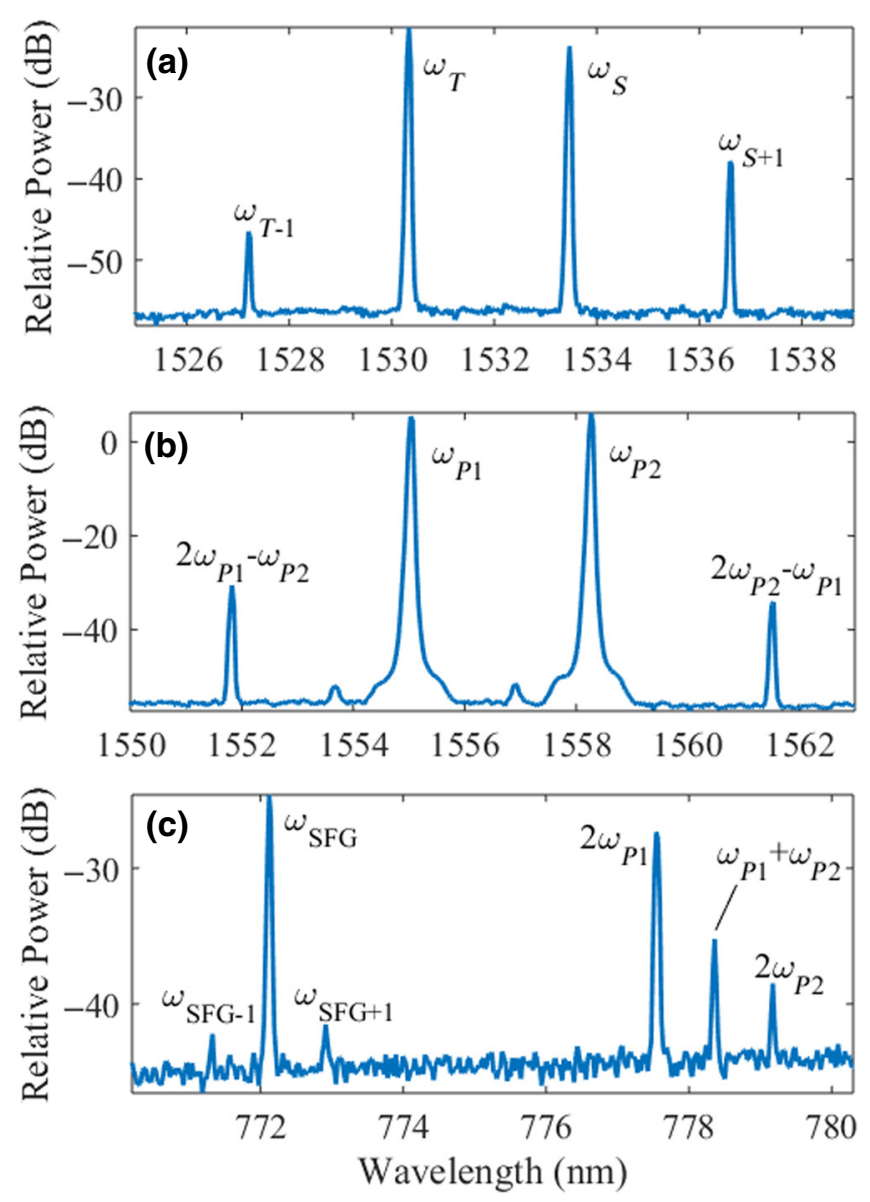

FIG. 4. Spectra from the output of the waveguide. (a) A spectrum around the signal and target wavelength. Two other peaks are present, generated by the interaction of the signal and idler with the wrong pumps. These peaks are at least $10 \mathrm{~dB}$ smaller than the correct ones. (b) The pump spectral region. The other peaks present are generated by DFG between the pumps and their own sum frequencies and second harmonics. (c) Spectra in the SFG region. Several parasitic nonlinear processes can be seen, with wrong-pump peaks occurring on either side of $\omega_{\mathrm{SFG}}$ and SFG/SHG interactions between the pumps to the right. 


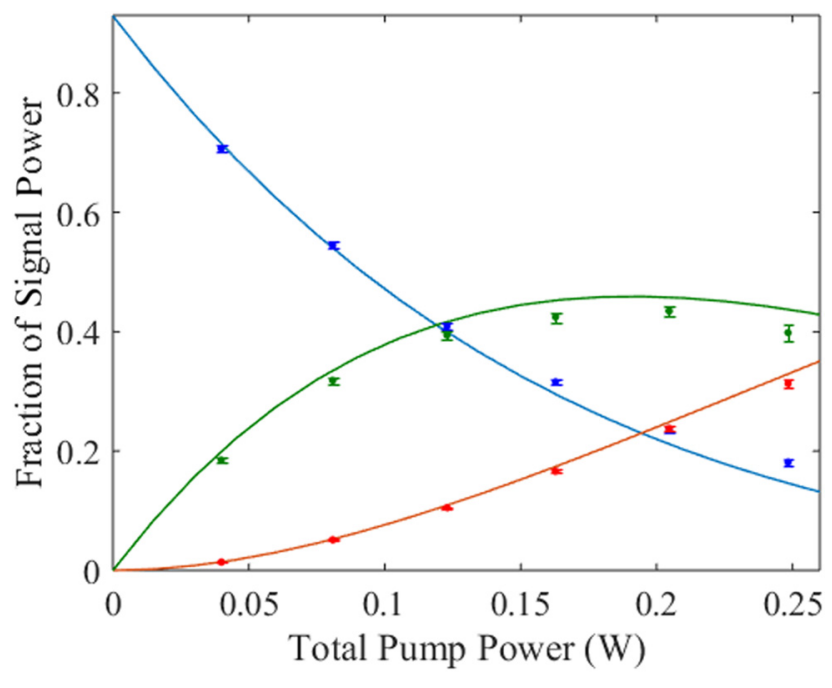

FIG. 5. Frequency conversion as a function of the total pump power for the signal (blue), SFG (green), and the target (red). The dots are experimental measurements while the solid lines are from theoretical calculations. The pump powers for $P_{1}$ and $P_{2}$ are balanced according to Eq. (7). The power values are measured out of the waveguide and are corrected for losses in the measurement setup.

output of the waveguide for up to a total pump power of $250 \mathrm{~mW}$ are shown in Fig. 5 (for the data at all wavelengths, see the Supplemental Material [27]). The values are corrected for losses in the measurement setup, including a $13 \%$ reflection loss at the output facet. The markers represent the relative power levels of the signal, target, and SFG while the solid lines are calculated from the theory. From this trend, it is estimated that maximum conversion requires $740 \mathrm{~mW}$ total pump power for a conversion efficiency $\eta_{c}=0.998$ and $\eta_{d}=1-1.02 \times 10^{-6}$. If the device was performing to the specified design without fabrication errors [31], using the whole $5 \mathrm{~cm}$ of poling and with an effective area of $25 \mu \mathrm{m}^{2}$, maximum conversion would only require $180 \mathrm{~mW}$ total pump power for a conversion efficiency $\eta_{c}=0.993$ and $\eta_{d}=1-1.22 \times 10^{-5}$.

\section{CONCLUSION}

By cascading $\chi^{(2)}$ nonlinear optical processes, we can achieve conversion between arbitrary frequencies and also overcome the speed bottleneck produced by a measureand-retransmit protocol. Our analysis of the role of phase mismatch allows us to improve upon other nonlinear optical schemes in terms of tuning bandwidth and efficiency. In a device with a single fixed poling period, there is an optimal choice of pumps that minimizes the average phase mismatch for any given conversion and maximizes the overall efficiency. Furthermore, in lithium niobate, the use of pumps near $2.38 \mu \mathrm{m}$ is both more efficient on average and leaves the entire $C$ band free from pumps and pump noise.

Our experimental results illustrate that this technique is quite feasible considering the maturity of our chosen platform of reverse-proton-exchanged waveguides in lithium niobate. Regardless, our formulation of this frequencyconversion technique applies to any $\chi^{(2)}$ nonlinear material or waveguide platform. An alternative, such as etched waveguides in thin-film lithium niobate, may provide the technology necessary to achieve longer interaction lengths more reliably.

\section{ACKNOWLEDGMENTS}

This work was supported by the Australian Research Council (ARC) Centre of Excellence for Quantum Computation and Communication Technology (Grant No. CE170100012) and the Griffith University Research Infrastructure Program. M.L. was supported by the Australian Research Council (ARC) Future Fellowship (Grant No. FT180100055). P.F. and M.V. were supported by the Australian Government Research Training Program Scholarship. This work was performed in part at the Queensland node of the Australian National Fabrication Facility, a company established under the National Collaborative Research Infrastructure Strategy to provide nano- and microfabrication facilities for Australia's researchers.

[1] ITU-T, Series G.694.1, type Tech. Rep. (Institution International Telecommunication Union, Geneva, 2012).

[2] Louay Eldada, Advances in ROADM technologies and subsystems, Proc. SPIE 5970, 597022 (2005).

[3] Chuan Pu, L. Y. Lin, E. L. Goldstein, and R. W. Tkach, Client-configurable eight-channel optical add/drop multiplexer using micromachining technology, IEEE Photonics Technol. Lett. 12, 1665 (2000).

[4] E. J. Klein, D. H. Geuzebroek, H. Kelderman, Gabriel Sengo, N. Baker, and A. Driessen, Reconfigurable optical add-drop multiplexer using microring resonators, IEEE Photonics Technol. Lett. 17, 2358 (2005).

[5] K. Inoue, Tunable and selective wavelength conversion using fiber four-wave mixing with two pump lights, IEEE Photonics Technol. Lett. 6, 1451 (1994).

[6] C. J. McKinstrie, J. D. Harvey, S. Radic, and M. G. Raymer, Translation of quantum states by four-wave mixing in fibers, Opt. Express 13, 9131 (2005).

[7] Alex S. Clark, Shayan Shahnia, Matthew J. Collins, Chunle Xiong, and Benjamin J. Eggleton, High-efficiency frequency conversion in the single-photon regime, Opt. Lett. 38, 947 (2013).

[8] Qing Li, Marcelo Davanço, and Kartik Srinivasan, Efficient and low-noise single-photon-level frequency conversion interfaces using silicon nanophotonics, Nat. Photonics 10, 406 (2016).

[9] Carsten Langrock, Eleni Diamanti, Rostislav V. Roussev, Yoshihisa Yamamoto, M. M. Fejer, and Hiroki Takesue, 
Highly efficient single-photon detection at communication wavelengths by use of upconversion in reverse-protonexchanged periodically poled $\mathrm{LiNbO}_{3}$ waveguides, Opt. Lett. 30, 1725 (2005).

[10] R. Osellame, R. Ramponi, M. Marangoni, G. Tartarini, and P. Bassi, Integrated all-optical nonlinear device for reconfigurable add/drop and wavelength shifting of WDM signals, Appl. Phys. B 73, 505 (2001).

[11] Yu Song and Gu Wanyi, A tunable wavelength conversion and wavelength add/drop scheme based on cascaded second-order nonlinearity with double-pass configuration, IEEE J. Quantum Electron. 41, 1007 (2005).

[12] Katia Gallo, Gaetano Assanto, and George I. Stegeman, Efficient wavelength shifting over the erbium amplifier bandwidth via cascaded second order processes in lithium niobate waveguides, Appl. Phys. Lett. 71, 1020 (1997).

[13] M. H. Chou, I. Brener, M. M. Fejer, E. E. Chaban, and S. B. Christman, 1.5- $\mu \mathrm{m}$-band wavelength conversion based on cascaded second-order nonlinearity in $\mathrm{LiNbO}_{3}$ waveguides, IEEE Photonics Technol. Lett. 11, 653 (1999).

[14] Katia Gallo and Gaetano Assanto, Analysis of lithium niobate all-optical wavelength shifters for the third spectral window, J. Opt. Soc. Am. B. 16, 741 (1999).

[15] Chen Bo and Xu Chang-Qing, Analysis of novel cascaded $\chi^{(2)}(\mathrm{SFG}+\mathrm{DFG})$ wavelength conversions in quasi-phasematched waveguides, IEEE J. Quantum Electron. 40, 256 (2004).

[16] Yong Wang and Chang-Qing Xu, Analysis of picosecondpulse wavelength conversion based on cascaded sumfrequency generation and difference-frequency generation in quasi-phase-matched $\mathrm{LiNbO}_{3}$ waveguides, Opt. Eng. 46, 055003 (2007).

[17] Bing Zhou, Chang-Qing $\mathrm{Xu}$, and Bo Chen, Comparison of difference-frequency generation and cascaded $\chi^{(2)}$ based wavelength conversions in $\mathrm{LiNbO}_{3}$ quasi-phase-matched waveguides, J. Opt. Soc. Am. B. 20, 846 (2003).

[18] J. Yamawaku, A. Takada, E. Yamazaki, O. Tadanaga, H. Miyazawa, and M. Asobe, in Conference on Lasers and Electro-Optics (Optical Society of America, Baltimore, Maryland, United States, 2003), p. 1135.

[19] Y. Min, J. Lee, Y. Lee, W. Grundkoetter, V. Quiring, and W. Sohler, in Optical Fiber Communications Conference (IEEE, Atlanta, Georgia, United States, 2003), Vol. 2, p. 767.

[20] H. Furukawa, A. Nirmalathas, N. Wada, S. Shinada, H. Tsuboya, and T. Miyazaki, Tunable all-optical wavelength conversion of $160-\mathrm{GB} / \mathrm{s}$ RZ optical signals by cascaded SFG-DFG generation in PPLN waveguide, IEEE Photonics Technol. Lett. 19, 384 (2007).
[21] Yeung Lak Lee, Changsoo Jung, Young-Chul Noh, Mahn Yong Park, Clare C. Byeon, Do-Kyeong Ko, and Jongmin Lee, Channel-selective wavelength conversion and tuning in periodically poled $\mathrm{Ti}: \mathrm{LiNbO}_{3}$ waveguides, Opt. Express 12, 2649 (2004).

[22] Guo-Wei Lu, Satoshi Shinada, Hideaki Furukawa, Naoya Wada, Tetsuya Miyazaki, and Hiromasa Ito, 160-GB/s alloptical phase-transparent wavelength conversion through cascaded SFG-DFG in a broadband linear-chirped PPLN waveguide, Opt. Express 18, 6064 (2010).

[23] Yeung Lak Lee, Changsoo Jung, Young-Chul Noh, Il Woo Choi, Do-Kyeong Ko, Jongmin Lee, Han-Young Lee, and Hubertus Suche, Wavelength selective single and dual-channel dropping in a periodically poled $\mathrm{Ti}: \mathrm{LiNbO}_{3}$ waveguide, Opt. Express 12, 701 (2004).

[24] Yeung Lak Lee, Bong-Ahn Yu, Changsoo Jung, YoungChul Noh, Jongmin Lee, and Do-Kyeong Ko, All-optical wavelength conversion and tuning by the cascaded sumand difference frequency generation (cSFG/DFG) in a temperature gradient controlled Ti:PPLN channel waveguide, Opt. Express 13, 2988 (2005).

[25] Miloš Rančić, Morgan P. Hedges, Rose L. Ahlefeldt, and Matthew J. Sellars, Coherence time of over a second in a telecom-compatible quantum memory storage material, Nat. Phys. 14, 50 (2017).

[26] Chaitali Joshi, Alessandro Farsi, Stéphane Clemmen, Sven Ramelow, and Alexander L. Gaeta, Frequency multiplexing for quasi-deterministic heralded single-photon sources, Nat. Commun. 9, 847 (2018).

[27] See the Supplemental Material at http://link.aps.org/supple mental/10.1103/PhysRevApplied.13.024017 for the theoretical derivation and further experimental data.

[28] G. J. Edwards and M. Lawrence, A temperature-dependent dispersion equation for congruently grown lithium niobate, Opt. Quantum Electron. 16, 373 (1984).

[29] Francesco Lenzini, Sachin Kasture, Ben Haylock, and Mirko Lobino, Anisotropic model for the fabrication of annealed and reverse proton exchanged waveguides in congruent lithium niobate, Opt. Express 23, 1748 (2015).

[30] Sachin Kasture, Francesco Lenzini, Ben Haylock, Andreas Boes, Arnan Mitchell, Erik W. Streed, and Mirko Lobino, Frequency conversion between UV and telecom wavelengths in a lithium niobate waveguide for quantum communication with $\mathrm{Yb}^{+}$trapped ions, J. Opt. 18, 104007 (2016).

[31] Benjamin F. Johnston, Ph.D. thesis, MQ Photonics Research Centre, Department of Physics, Macquarie University, 2008. 